\title{
Putting concepts into context
}

\author{
Eiling Yee $^{1,2} \cdot$ Sharon L. Thompson-Schill ${ }^{3}$
}

Published online: 9 June 2016

(C) Psychonomic Society, Inc. 2015

\begin{abstract}
At first glance, conceptual representations (e.g., our internal notion of the object "lemon") seem static; we have the impression that there is something that the concept lemon "means" (a sour, yellow, football-shaped citrus fruit) and that this meaning does not vary. Research in semantic memory has traditionally taken this "static" perspective. Consequently, only effects demonstrated across a variety of contexts have typically been considered informative regarding the architecture of the semantic system. In this review, we take the opposite approach: We review instances of context-dependent conceptual activation at many different timescales - from long-term experience, to recent experience, to the current task goals, to the unfolding process of conceptual activation itself-and suggest that the pervasive effects of context across all of these timescales indicate that rather than being static, conceptual representations are constantly changing and are inextricably linked to their contexts.
\end{abstract}

Keywords Embodied cognition · Semantic memory

Imagine a chef, searching a basket of fresh produce for a lemon with a perfectly yellow peel. Now imagine that same chef, carefully peeling the lemon with a special

Eiling Yee

eiling.yee@gmail.com

1 Department of Psychology, University of Connecticut, 406 Babbidge Road, Unit 1020, Storrs, CT 06269-1020, USA

2 Basque Center on Cognition, Brain \& Language, Donostia, Spain

3 Department of Psychology, University of Pennsylvania, Philadelphia, PA, USA curved paring knife to form a "flower" garnish carved from the peel. When the chef thinks about a lemon in these two contexts, is she thinking about the same thing? Or does the information that she activates change depending upon the context? How constant, or "static," are conceptual representations across contexts? And how constant are they across different people, and across time? Is a well-trained chef's representation of a lemon, or a paring knife, different from yours or mine? One might suppose that semantic memory is essentially (1) "context-free" (surely a lemon has certain invariant properties the chef can rely on across contexts?), (2) independent of any specific event (isn't a lemon that the chef slices up essentially the same thing as one that she peels?), and (3) shared (how else could the chef communicate about a lemon if the concept were not shared?). However, we believe that to understand the semantic system, our theories must incorporate explanations of those aspects of semantic memory that are neither context-free, nor independent of specific events, nor shared.

Traditionally, to contrast semantic memory with the individual and specific experiences that make up episodic memory, psychologists and cognitive neuroscientists have indeed described semantic memory as "context-free." And conceptual representations (e.g., the concept of a lemon) do at first glance seem static - that is, we have the impression that there is something that the concept lemon "means" (a sour, yellow, football-shaped citrus fruit) and that this meaning does not vary, even when we think about it in different contexts.

However, current accounts of conceptual knowledge allow for the possibility that conceptual representations are instead more dynamic. In these models, object concepts are multidimensional representations that are distributed (in part) across brain regions that underlie sensory, perceptual, and motor functions (e.g., Allport, 1985; Barsalou, 1999; Damasio, 
1989). ${ }^{1}$ Thus, according to these "sensorimotor-based" accounts of semantic memory, the "meaning" of a lemon is not an indivisible whole, but is distributed across a range of featural dimensions (cf. McRae, de Sa, \& Seidenberg, 1997; Rogers \& McClelland, 2004; Tyler \& Moss, 2001; Vigliocco, Vinson, Lewis, \& Garrett, 2004). Because of this distributed architecture, there is no impediment to certain features of a concept being more active than others (e.g., depending on the circumstances). Thus, a distributed architecture allows conceptual representations to be dynamically sensitive to context (for discussions, see Humphreys \& Forde, 2001; Kiefer \& Martens, 2010; Schyns, Goldstone, \& Thibaut, 1998; Spivey, 2007).

Despite the fact that current models of semantic memory suggest that conceptual representations can be dynamic, many empirical investigations into semantic memory still focus on the invariance of conceptual representation; the implicit (or, sometimes, explicit) assumption is that only effects that can be demonstrated across a variety of contexts should be considered informative with regard to the structure and organization of semantic memory. Here we take a very different perspective - one in which context is instead a fundamental property of the structure of the semantic system.

Of course, no one would deny that context influences conceptual processing - decades of semantic priming studies have shown just this (e.g., Barclay, Bransford, Franks, McCarrell, \& Nitsch, 1974; Meyer \& Schvaneveldt 1971; Tabossi, 1988), and several authors have considered context effects and/or conceptual flexibility in the context of evaluating sensorimotor-based (or "embodied") theories of conceptual representation (e.g., Dove, 2015; Hauk \& Tschentscher, 2013; Kiefer \& Pulvermüller, 2012; Mahon, 2015) and language processing (Taylor \& Zwaan, 2009; Willems \& Casasanto, 2011). Our point is not simply that conceptual access changes with context. Instead, we contend that (as has been suggested in the context of traditional approaches to episodic memory; for a review, see Schacter, 1995) the concepts themselves are inextricably linked to the contexts in which they appear, so much so that the dividing line between a concept and a context may be impossible to clearly make out (e.g., Barsalou, 1987; Casasanto \& Lupyan, 2015; Connell \& Lynott, 2014b; Elman, 2004; Lebois, Wilson-Mendenhall, \& Barsalou, 2014; Prinz, 2002; Yee, Chrysikou, \& ThompsonSchill, 2013).

In this article, we review evidence that conceptual representations are intertwined with context. The contexts that we describe (which are likely interdependent) include: (1) longterm experience, (2) recent experience, (3) the concurrent

\footnotetext{
${ }^{1}$ We focus in this review on object concepts, but we believe that the principles that we discuss should apply to abstract concepts, too; in particular, we suspect that abstract concepts should be affected by context across multiple timescales.
}

context or ongoing goals, and (4) the passage of time as the process of object recognition unfolds. Although we believe that context should really be conceptualized in more continuous terms, for expository purposes we describe it at these four discrete timescales. We also discuss an additional factor, (5) individual abilities, which we hypothesize may modulate the role of context generally.

Finally, we will briefly review one theoretical framework in which all of these context effects are a natural consequence of how concepts are acquired and represented in the first place, and in which change over time is naturally accommodated. Although the context effects that we describe may be consistent with views in which context simply changes which aspects are activated of concepts that are fundamentally static, a static framework does not easily accommodate learning. Therefore, we suggest that a more parsimonious account is a dynamic framework in which a concept cannot be meaningfully separated from the context in which it is understood.

\section{Long-term experience}

Does our well-trained chef have different representations for special cooking implements than a culinary novice does? If she does, how did this come to be the case? It is generally accepted that the episodic memory system has a major role in acquiring semantic knowledge. But how does this acquisition process work? And is there a point at which our concepts of things stop changing, despite new episodes of experiencing them?

A fundamental prediction of sensorimotor-based theories is that an object's conceptual representation includes the sensorimotor regions that are routinely active when that object is perceived or interacted with. This means that experiences with objects should shape representations, and as experience changes over time, representations should change as well. Moreover, given that different individuals have different experiences, conceptual representations should differ across individuals. However, few studies in the semantic memory literature have examined how individual differences in, or changes over, long-term experience affect the representations of object concepts. In this first section, we review some exceptions-studies showing that experience with objects shapes their conceptual representations.

For instance, there is evidence that the manner of experience that one has had with an object-for example, which hand is typically used to interact with it-affects conceptual representations: In right-handers, naming pictures of tools activates left premotor cortex more than in left-handers (but critically, there is no laterality difference for nonmanipulable objects - e.g., animals; Kan, Kable, Van Scoyoc, Chatterjee, \& ThompsonSchill, 2006; see Willems, Hagoort, \& Casasanto, 2010, for an analogous finding when reading action verbs, such as 
write or throw). In addition to conceptual representations being affected by the manner of manual experience, they also appear to be affected by the amount of manual experience: In a study that demonstrated that performing a concurrent manual task can selectively interfere with thinking about manually experienced objects (Yee, Chrysikou, Hoffman, \& Thompson-Schill, 2013we describe this study further in the section on the concurrent context), we also showed that the amount of experience that an individual has had with an object predicts how much disruption will be experienced from the manual task. This suggests that manual experience shapes the conceptual representation of manually experienced objects. Similarly, ratings of lifetime tactile experience with objects have been shown to correlate with the degree to which a brain region involved in objectrelated action (left parietal cortex) becomes active when making judgments about the objects' shapes (Oliver, Geiger, Lewandowski, \& Thompson-Schill, 2009).

Other types of long-term experience can also affect conceptual representations. In professional musicians, for example, identifying pictures of musical instruments activates auditory association cortex and adjacent areas more than identifying pictures of other objects (but no such difference is observed for musical lay people; Hoenig et al., 2011). Analogous findings have been reported for experience playing sports: Reading sentences describing actions associated with ice hockey (but not sentences describing everyday experience) activates premotor regions more in ice hockey players than in nonplayers (Beilock, Lyons, Mattarella-Micke, Nusbaum, \& Small, 2008).

Even without years of training, differences in sensorimotor experience can produce differences in an object's conceptual representation. For instance, after participants were trained for three weeks to classify novel objects while either pointing at the objects or pantomiming actions toward them, participants who had pantomimed, but not those who had pointed showed early activity in motor regions and later activity in occipito-parietal visual-motor regions (Kiefer, Sim, Liebich, Hauk \& Tanaka, 2007). This was the case even though the task was simply to make a category judgment on the written names of the novel objects. This study and related ones (Oliver, Parsons, \& Thompson-Schill, 2008; Weisberg, Turennout, $\&$ Martin, 2007) suggest that even shorter-term differences in sensorimotor experience can have a measurable impact on an object's conceptual representation.

The findings that we have described in this section may seem problematic. If one's concept of, for instance, a lemon changes with long-term experience, then no two individuals' concepts of lemons will be exactly the same. And furthermore, your own concept of a lemon might change subtly (or, in some cases, less subtly) over time, without your being consciously aware of the change. Yet we contend that this is, in fact, what happens, and that (as we will argue later) this is not as much of a problem as it might at first seem.

\section{Recent experience}

We now turn to work suggesting that if our chef had recently been searching for a lemon among the limes in her produce basket, the way that she subsequently thinks about lemons (and other colorful things) would be different than if she had recently been squeezing lemons for lemonade. In other words, conceptual activation can be influenced by recent experience.

For instance, van Dantzig, Pecher, Zeelenberg, and Barsalou (2008) have shown that the modality to which attention is directed immediately prior to thinking about objects can affect conceptual activation: Between trials that required participants to make true-false judgments on sentences referring to object properties (e.g., broccoli is green or soup is hot), participants responded to either a visual light, an auditory noise, or a tactile vibration; property judgments were faster when the modality to which the sentence referred was the same as the preceding perceptual stimulus. Along similar lines, but using a more implicit measure of conceptual activation, Bermeitinger, Wentura, and Frings (2011) found that when an independent task directing attention to shape was interspersed with a semantic-priming task, priming for words referring to natural kinds (for which shape is known to be a particularly important feature) was greater than priming for artifacts. In contrast, priming was greater for artifacts (for which action is known to be a particularly important feature) than for natural kinds when the interspersed task directed attention to action. By interspersing a task that directs attention to one modality or another with a task that requires conceptual activation, these two studies show that directing attention to a particular modality changes subsequent conceptual activation such that information related to that modality is activated more easily (for related behavioral findings, see Martens, Ansorge, \& Kiefer, 2011; Van Dam, Rueschemeyer, Lindemann, \& Bekkering, 2010; for related functional magnetic resonance imaging [fMRI] findings, see Hoenig, Sim, Bochev, Herrnberger, \& Kiefer, 2008; Mummery, Patterson, Hodges, \& Price, 1998; Phillips, Noppeney, Humphreys, \& Price, 2002; Thompson-Schill, Aguirre, D'Esposito, \& Farah, 1999; Rogers, Hocking, Mechelli, Patterson, \& Price, 2005; Van Dam, van Dijk, Bekkering, \& Rueschemeyer, 2012; for a review, see Willems \& Francken, 2012).

Conceptual activation can also be influenced by whether one initially heard the sound associated with a concept versus its name. For example, hearing "cat" facilitates subsequent judgments about whether an image of a cat is upright or upside down relative to hearing the sound of a cat meowing or the 
word "meow" (Lupyan \& Thompson-Schill, 2012)—perhaps because thinking about the noise that something makes focuses attention on a relatively limited part of its conceptual representation (e.g., features relating to that noise), whereas hearing the label may produce broader activation (i.e., of multiple features, including those that support an orientation judgment).

Perhaps surprisingly, there is also evidence that the modality to which attention has recently been directed can have a more lingering influence (e.g., from a prior task, rather than just from a prior trial) on conceptual activation: Although lemons and daffodils overlap on the dimension of color (both are yellow), and might therefore be expected to partially activate one another due to having overlapping conceptual representations, the word lemon does not ordinarily prime daffodil. Yet, it can prime daffodil if participants' attention has been focused on color in a prior task involving unrelated items (e.g., color words in a Stroop task; Yee, Ahmed, \& Thompson-Schill, 2012). Similar findings have been reported in other modalities. For example, Pecher, Zeelenberg, and Raaijmakers (1998) observed shape priming (e.g., the word coin priming the word button) only when, prior to the priming experiment, participants had made shape judgments about the objects to which the words referred. Thus, recent experience can linger long enough to affect conceptual activation in a subsequent, unrelated task.

Simply having recently named a picture of an object can also affect its subsequent activation: Targets categorically related to primes that had been recently named (in a prescanning picture-naming task), produced more fMRI adaptation than targets related to primes that had not been recently named (Gotts, Milleville, \& Martin, 2015). One interpretation of this finding (related to what we suggested above regarding hearing an object named) is that labeling an object leads to a more abstract/modality-neutral instantiation of its representation than does simply viewing it, and that such instantiations tend to overlap more with categorically related items. But whatever the reason, prior identification influences conceptual activation several minutes later in a subsequent task.

The studies described in this section show that recent experience (whether it be immediately prior to conceptual activation or several minutes before) that directs attention to a particular modality, or to no modality in particular, can influence conceptual activation. One could interpret these findings as a more mundane demonstration of the effects of attention to different features of an otherwise static concept; however (as we will describe later, in the section on emergent representations), we subscribe to the view that it is more appropriate to think of the concept itself as changing slightly each time it is retrieved, and that there is no real demarcation between what is activated in a given instance and the concept itself.

\section{The concurrent context or ongoing goals (i.e., task)}

Perceptual properties such as shape and color are particularly relevant when searching for a specific object (e.g., a lemon in a basket of produce). But, as we alluded to in the introduction, in other tasks form may be less relevant, and function more so (e.g., when the goal is to cut the lemon). In this section, we describe some studies that have shown that the immediate context or current goals can influence the overall degree to which different types of conceptual features become active.

For example, there is evidence that the immediate visual context can affect conceptual activation: If an object is depicted in a context consistent with its use (e.g., a kitchen timer on the counter next to a pot of food bubbling on the stove), the action associated with using the object is more readily available than when the same object is depicted in a context consistent with picking it up (e.g., when the timer is in a kitchen drawer; Kalénine, Shapiro, Flumini, Borghi, \& Buxbaum, 2014). ${ }^{2}$ There is also neural evidence that surrounding objects can influence conceptual activation. In an fMRI study, Hsu, Kraemer, Oliver, Schlichting, and Thompson-Schill (2011) asked participants to judge which of two objects a third object most resembled in color. When the three objects were all from the same color category (e.g., butter, egg yolk, and a school bus are all yellow), and thus the task context required retrieving detailed color knowledge, the neural response overlapped more with brain regions involved in color perception (the left fusiform) than when two of the three objects were from different color categories (e.g., one red and two yellow objects), and thus less detailed color knowledge was necessary. This finding is particularly interesting because it implies that the degree of perceptual resolution required by the task may influence the extent to which brain areas that support perception are involved: A task that requires a high degree of perceptual resolution involves perceptual areas more than a task that can be performed on the basis of more categorical (or abstracted) knowledge. Thus, conceptual knowledge may be represented at multiple levels of abstraction (for discussion, see Binder \& Desai, 2011; Thompson-Schill, 2003). We return to this idea later.

Conceptual activation can also be affected by whatever else we happen to be doing at the same time (and this is not simply because multitasking is difficult). For instance, we have shown that performing a concurrent manual task that is

\footnotetext{
${ }^{2}$ The way the object is oriented can also be important: How an object is grasped for use can be activated by a picture of that object (e.g., a beer mug with a handle; Tucker \& Ellis, 1998), but only if the pictured object is oriented in a way that is consistent with the object's function (Masson, Bub, \& Breuer, 2011).
} 
incompatible with how a given object is acted upon can interfere with thinking about that object. ${ }^{3}$ That is, if, while naming pictures, participants had to concurrently perform a sequence of hand motions that were incompatible with those used to interact with frequently manipulated objects, picture naming was more disrupted for objects that are typically interacted with manually than for objects that are less frequently interacted with manually (e.g., there was relatively more interference for pencils than for tigers; Yee, Chrysikou, Hoffman, \& Thompson-Schill, 2013; see also Witt, Kemmerer, Linkenauger, \& Culham, 2010). Thus, the context of a concurrent manual task interfered with people's ability to access the manipulation features of objects. This demonstrates not only that manipulation information is part of the representation of frequently manipulated objects, but also that our ability to access a given object depends on the match between our mental representation of that object's meaning and what we are doing at the moment. We return to this issue later, when we consider the argument that (contrary to what these data suggest) sensorimotor information, rather than being part of a concept, is accessed only incidentally during conceptual retrieval (for discussion, see Chatterjee, 2010; Mahon \& Caramazza, 2008).

Moving beyond action, a concurrent task can also interfere with accessing knowledge about the visual or auditory properties of objects. While holding in memory a sequence of three auditory tones, participants had more trouble verifying an auditory property of an object (e.g., blenders can be loud) than a visual property (e.g., lemons can be yellow). Furthermore, the complementary pattern was observed when holding in mind a sequence of three meaningless visual shapes (Vermeulen, Corneille, \& Niedenthal, 2008).

Similarly, conceptual activation can be influenced by the relationship between the meaning of a given concept and the modality emphasized by the current task: In an elegant study, Connell and Lynott (2014a) showed that a task that implicitly focuses attention on the visual modality (visual lexical decision) facilitates access to words referring to properties or things that are frequently experienced visually (e.g., the concept corresponding to the term small is rated as being experienced more by seeing than is the concept corresponding to the term

\footnotetext{
${ }^{3}$ Note the important difference between procedures that lead to facilitation (such as those described in the section on recent experience) and those that lead to interference. When a prior perceptual or motor task draws a participant's attention to a given modality, but does not continue to place task demands on the participant during a subsequent conceptual task, access to conceptual information in that modality can be facilitated (because the task drew attention to the modality but is not occupying resources in that modality). In contrast, in the concurrent tasks we describe here, the perceptual or motor task is ongoing (and thus occupying resources) while the concept is being accessed. This leads to interference in cases in which the concurrent task taps resources that are involved in representing the concept in question (see Connell \& Lynott, 2012).
}

husky). In contrast, a task that implicitly focuses attention more on the auditory modality (reading aloud) facilitates access to words referring to properties or things that are more frequently experienced auditorily (e.g., the concept corresponding to the term husky is rated as being experienced more by hearing than is the concept corresponding to the term small).

The studies we have described in this section make clear that both ongoing goals (i.e., the task) and the concurrent context can influence conceptual activation (for a related discussion, see Connell \& Lynott, 2014b). These context effects suggest that conceptual representations are distributed across features such that different features can become more or less active in different circumstances. More broadly, because altering the task or goals routinely alters conceptual activation, the findings imply that there is in fact no such thing as taskindependent conceptual activation.

In other words, although in the lexical-processing and semantic-memory literatures context is usually regarded as a means of evoking a particular, stable sense of a word or concept (e.g., Swinney, 1979; Tabossi, 1988; Zwaan, Stanfield, \& Yaxley, 2002), in other domains, such as the study of episodic memory, context is understood as a continually varying signal that is always operating to affect cognition (e.g., Polyn, Norman, \& Kahana, 2009). That is, there is never "no context," or even "neutral context," as one might assume by reading the semantic-memory literature. Instead, we should consider the idea that concepts cannot be meaningfully separated from the contexts in which they appear.

One corollary of this last idea is that concepts that are associated with more similar contexts should have activation profiles that are more similar across contexts than do concepts associated with more dissimilar contexts. A recent fMRI study suggests that this is the case: A corpus-based measure of the diversity of the contexts in which a word appears in language (which can be taken as a measure of variability in conceptual representation) predicts the variability in the neural response to that word across three random presentation contexts. That is, words that, in corpora, appear in less diverse contexts (e.g., asparagus) elicit a less variable response across presentations than do words that appear in more diverse contexts (e.g., basket; Musz \& Thompson-Schill, 2015; cf. RodríguezFerreiro, Gennari, Davies, \& Cuetos, 2011). This result not only highlights that a word's neural representation is not static, but also that the representations of some words, and therefore the concepts to which they refer, may be more variable than others.

\section{The passage of time as object recognition unfolds}

Does a quick glance at a lemon (perhaps as you hurry through the produce section of the grocery store) result in a different lemon being activated than during a lingering look? Does the 
lemon being activated change as your lingering look proceeds? Our and others' work has suggested that the answer to both of these questions is "yes." That is, during the process of object recognition, conceptual activation changes dynamically, with different features becoming active at different rates.

For example, we have found evidence that during visual object identification, information about the form of an object (e.g., that knives are oblong) becomes available sooner than information about the function (i.e., about the purpose of use; e.g., that knives are used for cutting; Yee, Huffstetler, \& Thompson-Schill, 2011). Specifically, in a "visual-world" eyetracking study (Cooper, 1974; Tanenhaus, SpiveyKnowlton, Eberhard, \& Sedivy, 1995), we found that when participants were briefly exposed (for $1 \mathrm{~s}$ ) to an array of four objects and asked to click on the object corresponding to a heard word, they were sensitive to the fact that, at the conceptual level, one of the other objects in the display was similar in shape to the named object. For example, when participants heard the target word "Frisbee," they looked at a slice of pizza (another object that can be round). Importantly, shape similarity was not apparent in the visual depictions (e.g., a slice of pizza is triangular, a shape that a Frisbee cannot take); thus, the preferential fixations on the shape-related object were attributable to activation of conceptual shape information (and not to the current input to the senses).

However, with the same $1 \mathrm{~s}$ exposure to the array, we found no preferential fixations on objects related in function to the named object. For example, when participants heard the word "tape," they did not preferentially fixate on a bottle of glue. Yet when exposure to the array was lengthened to $2 \mathrm{~s}$ (the task was otherwise identical), we observed the opposite patternparticipants preferentially fixated on the function-related object, but not the shape-related object. Thus, these findings suggest that visual object recognition is a dynamically unfolding process in which function follows form.

Other studies using the visual-world paradigm have also shown that function information becomes active after other, arguably more perceptually available, aspects of conceptual knowledge. For instance, knowledge about the thematic relationships that an object can participate in (e.g., the knowledge that a broom is often paired with [and therefore seen with] a dustpan, or that a steak is paired/seen with a knife) and knowledge about an object's structural characteristics (its shape, size, and volume) appear to become available more rapidly and more transiently than information about the object's function (see Kalénine, Mirman, Middleton, \& Buxbaum, 2012, for thematic, and Lee, Middleton, Mirman, Kalénine, \& Buxbaum, 2013, for structural).

Future work will be needed to investigate the cause(s) of this chronology. One possibility is that because information about the purpose for which an object is used is not directly available via the senses, accessing this information requires more processing (and thus more time) than accessing perceptual information. Another possibility is that in the studies reviewed above, perceptual information had special status because the visual-world paradigm task - essentially a visual search task-requires attending to perceptual information. A third (compatible) possibility is that presenting stimuli in the visual modality (regardless of whether the task is visual search) places emphasis on visual information.

Semantic-priming studies using written words provide a hint that the modality of the stimulus, rather than the visual search task per se, plays a role in perceptual information's precedence. First, a few semantic-priming studies using written words have tested whether responses to a target word are facilitated when the target is preceded by a shape-related prime word. Although the results have been mixed (see the Pecher et al., 1998, study described in the section on recent experience), several studies have obtained evidence of shape priming (Flores d'Arcais, Schreuder, \& Glazenborg, 1985; Schreuder, Flores d'Arcais, \& Glazenborg, 1984). Intriguingly, these studies have also included prime-target pairs related on a more abstract dimension (similar to function/purpose of use; e.g., apple-banana or stapler-paperclip) and found evidence that priming based on this abstract/functional dimension emerges more reliably at long than short interstimulus intervals, whereas priming based on shape relatedness was larger at short than at long interstimulus intervals (Flores d'Arcais et al., 1985; Schreuder et al., 1984). In other words, the chronology parallels what has been observed in the visual world paradigm.

Along the same lines, when reading sentences referring to objects (e.g., a calculator), information that is directly available from perceiving the object (e.g., the grasp that one would use to pick up a calculator to move it) becomes less active over time, whereas information that requires more abstract knowledge about the purpose of the object (e.g., the finger poke that one would use to operate a calculator) becomes more active over time (Bub \& Masson, 2010).

Subscribers to a "static" model of conceptual representations might not agree that the reason that perceptual information becomes active early is because written words, like visual objects, place emphasis on the visual modality. Instead, they might interpret the findings that we have described so far as indicating that, irrespective of context, perceptual information becomes active first during conceptual activation (perhaps because it is dominant during the extremely frequent behavior of object recognition). However, as we describe next, when stimuli are presented in other modalities, perceptual information does not always become active first, suggesting that the stimulus modality indeed affects the dynamics of featural activation.

In a cross-modal semantic-priming study in which the primes were auditory words (and the targets were visual), priming for visually related targets was observed, but it was delayed in time relative to priming for targets related 
to the typical use of the prime ${ }^{4}$ (Moss, McCormick, \& Tyler, 1997). Another study that presented object names auditorily obtained analogous results-information that was directly available from viewing the object (here, information about how one grasped an object to move it) became active later and for a shorter duration than information about how an object was manipulated in order to use it - that is, its function (Bub \& Masson, 2012). Thus, in contrast to when primes are visual, when the primes (or the sole presented words) are auditory, the activation of perceptual information can appear later than that of functional information (see Garcea \& Mahon, 2012, for related work).

Taken together, the pattern across studies suggests that as the process of object recognition proceeds, some types of featural information become active earlier than others, and may also decay more quickly. Moreover, the ebb and flow of different features seems to be influenced by the relationship between the modality of the stimulus and the specific feature: When the stimulus is presented visually, visual features may become active earlier than more abstract knowledge (e.g., relating to purpose or use). In contrast, when the stimulus is presented auditorily, the reverse pattern has been observed. ${ }^{5}$ Thus, the dynamics of featural activation during object recognition appear to depend on the modality (i.e., the context) in which the concept is presented. This, together with evidence (reviewed in the first three sections) that conceptual activation is affected by long-term experience, recent experience, and current goals, suggests that rather than being fixed, concepts change from one context to another.

\section{Individual abilities}

So far, we have described evidence demonstrating that context can have an influence on conceptual activation at many different timescales (which we have suggested are probably better conceptualized in more continuous terms). However, we have not yet considered (1) whether factors intrinsic to the individual, such as individual differences in cognitive abilities/processing styles might impact an individual's conceptual processing in general, or (2) whether such factors might impact the extent to which a given individual is sensitive to context. In this section, we speculate about some

\footnotetext{
${ }^{4}$ The perceptually related targets were primarily visible parts of the prime or what the prime is typically made of (e.g., blouse-button or sandalleather), whereas the use-related targets typically denoted the primary purpose of the prime or the location at which the prime is used (e.g., blouse-wear, satchel-school, or radio-music).

${ }^{5}$ Task may also interact with which features become available when: Rogers and Patterson (2007) have shown that when the task is categorization, perceptual information seems to become available later than more general information.
}

individual factors that we hypothesize may affect both conceptual processing and the influence of context on such processing.

First, we consider whether individual differences in processing preferences (e.g., preferring to process stimuli visually vs. verbally) might impact conceptual activation. For instance, the same study that demonstrated that retrieving detailed color knowledge about objects produces activity in color perception brain regions (Hsu et al., 2011) also reported a correlation between activity in a color perception brain region and the extent to which participants reported preferring to process information more visually (relative to verbally, as measured by a questionnaire; Kirby, Moore, \& Schofield, 1988). Similarly, Kraemer, Rosenberg, and Thompson-Schill (2009) found that given a similarity judgment task that could be completed either by remembering a written list of features (e.g., a red, striped circle) or by converting that list into a visual image of the object, participants who reported a more verbal cognitive style showed more activity in a brain region associated with phonological processing (supramarginal gyrus), whereas participants who reported a more visual style showed more activity in a brain region associated with visual object processing (fusiform gyrus). These findings suggest that conceptual processing may be influenced by individual preferences to attend to one modality versus another.

Another study that we have already described (in the section on the concurrent context) is also consistent with the hypothesis that individual differences in processing preferences may affect conceptual activation. In the semantic-priming study in which lemon only primed daffodil if participants' attention had been focused on color in a prior, ostensibly unrelated Stroop task, we observed that individual differences in the ability to selectively focus on color in the Stroop task predicted the amount of priming. This relationship could reflect differences in the degrees to which people attend to or perceive color (as in Hsu et al., 2011): Individuals who attend to color more would more strongly associate conceptual color with both color words (e.g., green) and with the names of objects (e.g., cucumber).

However, another compatible possibility relates to selective attention: The general ability to selectively attend to one dimension at the expense of others (e.g., to focus on a word's font color while ignoring its meaning) is an aspect of cognitive control (Posner \& Snyder, 1975) and varies across individuals. Thus, a high capacity for selective attention could manifest as enhanced selective attention to the features most relevant for the current task (in this case, judging animal status), and hence as less activation of other features (e.g., color).

This account is particularly interesting because it is consistent with the proposal that cognitive control regulates the ability to selectively attend to the task-relevant features of a concept in general (see Kan \& Thompson-Schill, 2004b; Thompson-Schill, Bedny, \& Goldberg, 2005). Evidence consistent with this account has come from a recent study 
demonstrating that inhibitory electrical stimulation over left prefrontal cortex (a brain region that supports cognitive control) interferes with the ability to categorize objects according to a specific attribute (e.g., "round or red things") relative to categorizing objects at a more general level (e.g., "things that hold water"; Lupyan, Mirman, Hamilton, \& Thompson-Schill, 2012; for related work, see also Chrysikou et al., 2013; Kan \& ThompsonSchill, 2004a; Lupyan \& Mirman, 2013).

If individual processing preferences or cognitive control abilities indeed affect the extent to which conceptual activation is affected by context, then concepts must vary, not only from one context to another, but also within a given context, from one person to another. This underscores that concepts cannot be meaningfully separated from the contexts (including the individuals) in which they are understood.

\section{Emergent representations and contextually bound concepts}

In the preceding sections, we focused on how conceptual representations and their activation can be affected by longterm experience, by recent experience and the concurrent context or ongoing task, by the passage of time as object recognition unfolds, and by individual differences in cognitive function. At first glance, these may seem like disparate influences, and a full account of how these are in fact part of the same contextual dynamic is beyond the scope of this article. However, below, we will briefly review an approach to the development of conceptual representations in which there is little theoretical separation between these influences (with the exception of individual abilities, which we address later). This approach helps explain how, in the face of different experiences, individuals are able to understand each other when they use conventionalized labels (e.g., your meaning of "kite" is not so different from mine, even if you are a kite surfer and I have never been out on water). It also naturally accommodates learning, and along the way, explains why conceptual representations are inherently "fuzzy," with no context-free "core." The account leads to many of the same properties of a conceptual system that are outlined by Connell and Lynott (2014b) and is compatible with related, more neurally oriented accounts (e.g., Binder \& Desai, 2011; Damasio, 1989; Patterson, Nestor, \& Rogers, 2007; Pulvermüller, 1999; Rogers \& McClelland, 2004) and with earlier cognitive accounts (e.g., Malt \& Johnson, 1992; McCloskey \& Glucksberg, 1979). However, it adds a mechanistic explanation for how conceptual organization becomes the way it is.

Within the emergentist approach to cognitive development (e.g., Elman et al., 1996) and conceptual representations (e.g., Rogers \& McClelland, 2004), long-term knowledge is an emergent representation abstracted across multiple experiences. In one implementation of a small component of development, Elman $(1990,1993)$ showed how knowledge about, for example, a word (although the same principles can apply to a concept) could be conceived of as the accumulation of knowledge about the contexts in which that word can occur. A key point is that this type of model "keeps track" of systematic variation in the input, such that idiosyncratic things that happen when in the presence of a lemon (such as a dog barking, or a cloud moving overhead) would have minimal impact on its developing representation, whereas things that happen more systematically in the presence of lemons (e.g., if in most of your experiences with lemons you squeeze them) would be more likely to become part of the representation. Thus, long-term knowledge would be stored in a substrate that would gradually change with experience (see Altmann, 1997, for details of how the learning process in Elman [1990, 1993] could lead a concept to be abstracted across experiences).

In this model, there is no representational distinction between knowledge of something and knowledge of the contexts in which that thing occurs. In other words, whereas some accounts distinguish between types (or "concepts") and tokens (the instantiation of a concept in a particular episode), the emergentist account distinguishes these only to the extent that one (types) is the abstraction, across multiple experiences, of the other (tokens).

A critical insight implemented in these early emergentist models was that the state of the cognitive system at any one moment in time is a function of its accumulated experience, its current input (whatever in the environment attention is directed toward), and information about consecutively prior internal states of the cognitive system (via recurrence across time). The benefit of recurrence is that it allows for sequencing-for example, the knowledge we have about lemons that they should be cut before squeezing. (It also allows for abstraction over sequences, as is required in language acquisition, where language is an inherently sequenced input.) Thus, a recurrent architecture allows structure to be encoded across time.

Within such a framework, conceptual activation is simply the flow of activation (both excitatory and inhibitory) through a network of connections that cumulatively reflect prior experience. This notion of conceptual activation explains the contextual dynamics we have discussed in the preceding sections: Long-term experience not only gradually organizes the substrate in which knowledge is represented, it also influences the flow of activation due to the current input, just as shorter-term contextual experience also influences that flow. The concurrent context and task goals influence the input either directly (the concurrent context is the input, if not in the current time, in preceding moments of time that have already changed the internal state of the system) or indirectly, via top-down control of information 
flow (see Cohen, Aston-Jones, \& Gilzenrat, 2004, for an example of task-directed "gating" in a similar framework).

An important property of dynamical models with recurrence through time is that the input is not apprehended in a single "step," but rather unfolds across time (cf. the section on the passage of time as object recognition unfolds) - both as a property of the signal, if it changes across time (e.g., speech), and, critically, as a property of the internal dynamics of such systems. Even for inputs that do not appear to change across time (e.g., when apprehending a slice of pizza), the connectivity between regions of the network will mean that different parts of it (encoding, e.g., shape or function) will likely become activated at different times as a function of internal network dynamics, the temporal properties of the input, and top-down control. Within this framework, the kinds of individual differences that we describe in the previous section could arise when the control of information flow through one part of the network or another is more or less tightly regulated, perhaps as a function of "cognitive control" (e.g., Cohen et al., 2004; Frith, 2000; Mechelli, Price, Friston, \& Ishai, 2004; Miller \& Cohen, 2001; Noppeney, Price, Friston, \& Penny, 2006).

More generally, this dynamical framework predicts that conceptual knowledge, categories, and "types" can be no more invariant than the contexts over which they have been abstracted - and thus, contrary to the traditional definition of semantic memory as context-free, the dynamical framework blurs the distinction between the content of concepts and their context. Yet the framework also predicts that, to the extent that people can share experiences (e.g., seeing kites in the sky), they can also share labels for those experiences (my label "kite" is the same as your label "kite"). Critically, although many experiences are shared, others are distinctive, with the former enabling a common labeling system, and the latter producing different conceptual systems across individuals.

\section{Sensorimotor information: part of, or peripheral to, concepts?}

We return, finally, to an issue first raised in the section on the concurrent context: Is sensorimotor information a constituent part of the representations of concrete objects? It is worth noting that in the emergentist framework outlined in the preceding section, not only is there no distinction between the knowledge of what something is and the contexts in which that thing has occurred, there is also no distinction between some "core" part of the knowledge of something and some other, more "peripheral" part. Knowledge differs only insofar as it captures different contingencies between individual experiences and the generalizations formed on the basis of those individual experiences. Thus, in such a framework, the distinction between "part of" versus "incidental to" is not meaningful (Elman, 2009). Nevertheless, because this is a persistent issue within the semantic memory literature, we consider the evidence below.

Numerous studies (some reviewed above) have demonstrated that thinking about a concrete object produces activation in brain regions putatively involved in action upon or perceiving that object. But there is general agreement that, in some theoretical models at least (although not in the emergentist approach), such activations could, in principle, be incidental to the activation of an amodal (or "disembodied") concept, rather than part of it (for discussions, see Anderson \& Spivey, 2009; Chatterjee, 2010; Mahon \& Caramazza, 2008). One often-cited fact that has been pointed to as evidence that such activations are only incidental is that some patients with motor or sensory deficits, despite having difficulty performing, for example, object-related actions, can retain the ability to name, and may also be able to describe the use of objects with strongly associated actions. Such intact abilities have been taken as evidence that such individuals have intact conceptual knowledge, and thus that sensory or motor information is not part of conceptual knowledge (see Negri et al., 2007).

However, as we reviewed in the introduction, sensoriomotor-based models of semantic memory posit that conceptual representations can include many different components (e.g., visual, auditory, and olfactory, as well as actionoriented and multimodal) that are distributed across the cortex. Moreover, conceptual information may be represented at multiple levels of abstraction, and thus, depending on the context, conceptual activation may involve the activation of some levels more than others (Hsu et al., 2011). For these reasons (as we and others - e.g., Taylor \& Zwaan, 2009- have described previously), having difficulty accessing part of a representation would not be expected to result in catastrophic conceptual loss (although, depending on the task, some impairment might be detectable)_-just as losing one finger does not entail losing use of the entire hand. In other words, "brain damage leading to problems performing an action with a particular object does not entail difficulty recognizing that object . . . the object may be recognizable on the basis of other aspects of its representation (and the extent to which there are other aspects to rely upon may vary across individuals)" (Yee, Chrysikou, Hoffman, \& Thompson-Schill, 2013, pp. 917-918). Indeed, this is one of the theoretical advantages of a parallel-distributed-systems approach (McClelland, Rumelhart, \& the PDP Research Group, 1986) such as that described in the preceding section.

In fact, even access to information that is specifically related to the affected modality may be delayed rather than entirely lost (e.g., Lee, Mirman, \& Buxbaum, 2014; Myung et al., 2010)- a difference that would be difficult to detect at the relatively coarse grain of a naming, matching, or description task. Nevertheless, many studies have observed that, as predicted by sensorimotor-based models, conceptual information 
related to the affected modality is more difficult to access when sensorimotor systems are impaired or interfered with in some way-for example, via neural stimulation (Pobric, Jefferies, \& Lambon Ralph, 2010), a concurrent task (e.g., Yee, Chrysikou, Hoffman, \& Thompson-Schill, 2013), or brain damage (e.g., the delays observed in Lee et al., 2014, and Myung et al., 2010; or impaired naming, as in Herrera, Rodríguez-Ferreiro, \& Cuetos, 2012, and Trumpp, Kliese, Hoenig, Haarmeier, \& Kiefer, 2013; for a review, see Gainotti, 2000).

\section{Summary}

Collectively, the studies we have reviewed suggest that conceptual representations are fluid, changing not only as a function of context as it relates to stimulus modality and task, but also as a function of the context brought by a particular individual - via recent or long-term experience, or even via neural degeneration, processing preferences, or abilities.

We contend that the various changes that concepts can undergo in all of these contexts are not "noise" to be controlled away, but rather provide important clues about the architecture of the semantic system. Putting all of these dynamic effects together creates a (moving) picture that is naturally accommodated by a semantic system that is (1) experience-based, such that it allows representations to change over time, and (2) distributed, such that various features can become active at different rates in different circumstances.

It may seem that if what is retrieved from semantic memory is so variable across time and individuals, communication should be difficult. However, as we noted in the section on emergent representations, the significant commonalities in human experience (and especially within a given culture) would lead different individuals' representations (and their labels) to be similar enough for most practical purposes. Moreover, how often does communication require that the interlocutors be activating the exact same conceptual representations (for further consideration of this point, see Casasanto \& Lupyan, 2015; Connell \& Lynott, 2014b; Taylor \& Zwaan, 2009)? In fact, given that communication often requires clarification ("no, no, no ... that's not a beet, it's a turnip!"6), it should not be difficult to come to terms with the idea that concepts can differ across individuals.

Finally, we contend that the features over which we have described context exerting its influence are not arbitrary. Given that the dimensions over which we experience objects (and events, and actions) are constrained by our senses, it is

\footnotetext{
${ }^{6}$ Or a more apt example might be, "no, no, no, that's not a concept, that's a . .." And, indeed, abstract concepts may be more susceptible to communication failures, because when there is nothing concrete to point to, it is less clear when labels need to be adjusted.
}

inevitable that our concepts are constructed via experiencing objects through (and integrating across) these senses. Importantly, however, this is not to say that the content of the conceptual system is purely sensory: Abstraction - that is, representing higher-order contingencies across time and, critically, across contexts - is a critical aspect of the semantic system. And abstraction must occur across experiences at multiple levels and across multiple timescales. Thus, concepts, as abstractions, necessarily change as contexts change. In other words, just as our interactions with objects in the world are defined by the contexts in which they are embedded, so is the conceptual system in which these objects are represented.

Author Note We thank Gerry Altmann and Katherine White for helpful discussion.

\section{References}

Allport, D. A. (1985). Distributed memory, modular subsystems and dysphasia. In S. K. Newman \& R. Epstein (Eds.), Current perspectives in dysphasia (pp. 207-244). Edinburgh, UK: Churchill Livingstone.

Altmann, G. T. M. (1997). The Ascent of Babel: An exploration of language, mind, and understanding. Oxford, UK: Oxford University Press.

Anderson, S. E., \& Spivey, M. J. (2009). The enactment of language: Decades of interactions between linguistic and motor processes. Language and Cognition, 1, 87-111.

Barclay, J. R., Bransford, J. D., Franks, J. J., McCarrell, N. S., \& Nitsch, K. E. (1974). Comprehension and semantic flexibility. Journal of Verbal Learning and Verbal Behavior, 13, 471-481.

Barsalou, L. W. (1987). The instability of graded structure: Implications for the nature of concepts. In U. Neisser (Ed.), Concepts and conceptual development (pp. 101-140). Cambridge, UK: Cambridge University Press.

Barsalou, L. W. (1999). Perceptual symbol systems. Behavioral and Brain Sciences, 22, 577-609. doi:10.1017/S0140525X99002149. disc. 609-660.

Beilock, S. L., Lyons, I. M., Mattarella-Micke, A., Nusbaum, H. C., \& Small, S. L. (2008). Sports experience changes the neural processing of action language. Proceedings of the National Academy of Sciences, 105, 13269-13273.

Bermeitinger, C., Wentura, D., \& Frings, C. (2011). How to switch on and switch off semantic priming effects for natural and artifactual categories: Activation processes in category memory depend on focusing specific feature dimensions. Psychonomic Bulletin \& Review, 18, 579-585. doi:10.3758/s13423-011-0067-z

Binder, J. R., \& Desai, R. H. (2011). The neurobiology of semantic memory. Trends in Cognitive Sciences, 15, 527-536. doi:10.1016/ j.tics.2011.10.001

Bub, D. N., \& Masson, M. E. J. (2010). On the nature of hand-action representations evoked during written sentence comprehension. Cognition, 116, 394-408.

Bub, D. N., \& Masson, M. E. J. (2012). On the dynamics of action representations evoked by names of manipulable objects. Journal of Experimental Psychology: General, 141, 502-517. doi:10.1037/ a0026748

Casasanto, D., \& Lupyan, G. (2015). All concepts are ad hoc concepts. In E. Margolis \& S. Laurence (Eds.), The conceptual mind: New 
directions in the study of concepts (pp. 543-566). Cambridge, MA: MIT Press.

Chatterjee, A. (2010). Disembodying cognition. Language and Cognition, 2, 79-116. doi:10.1515/LANGCOG.2010.004

Chrysikou, E. G., Hamilton, R. H., Coslett, H. B., Datta, A., Bikson, M., \& Thompson-Schill, S. L. (2013). Non-invasive transcranial direct current stimulation over the left prefrontal cortex facilitates cognitive flexibility in tool use. Cognitive Neuroscience, 4, 81-89.

Cohen, J. D., Aston-Jones, G., \& Gilzenrat, M. S. (2004). A systems-level perspective on attention and cognitive control: Guided activation, adaptive gating, conflict monitoring, and exploitation vs. exploration. In M. I. Posner (Ed.), Cognitive neuroscience of attention (pp. 71-90). New York, NY: Guilford Press.

Connell, L., \& Lynott, D. (2012). When does perception facilitate or interfere with conceptual processing? The effect of attentional modulation. Frontiers in Psychology, 3, 474. doi:10.3389/fpsyg.2012. 00474

Connell, L., \& Lynott, D. (2014a). I see/hear what you mean: Semantic activation in visual word recognition depends on perceptual attention. Journal of Experimental Psychology: General, 143, 527-533.

Connell, L., \& Lynott, D. (2014b). Principles of representation: Why you can't represent the same concept twice. Topics in Cognitive Sciences, 6, 390-406. doi:10.1111/tops.12097

Cooper, R. M. (1974). The control of eye fixation by the meaning of spoken language: A new methodology for the real-time investigation of speech perception, memory, and language processing. Cognitive Psychology, 6, 84-107.

Damasio, A. R. (1989). The brain binds entities and events by multiregional activation from convergence zones. Neural Computation, 1, $123-132$

Dove, G. (2015). Three symbol ungrounding problems: Abstract concepts and the future of embodied cognition. Psychonomic Bulletin \& Review. Advance online publication. doi:10.3758/s13423-0150825-4

Elman, J. L. (1990). Finding structure in time. Cognitive Science, 14, $179-211$

Elman, J. L. (1993). Learning and development in neural networks: The importance of starting small. Cognition, 48, 71-99.

Elman, J. L. (2004). An alternative view of the mental lexicon. Trends in Cognitive Sciences, 8, 301-306. doi:10.1016/j.tics.2004.05.003

Elman, J. L. (2009). On the meaning of words and dinosaur bones: Lexical knowledge without a lexicon. Cognitive Science, 33, 547582. doi:10.1111/j.1551-6709.2009.01023.x

Elman, J. L., Bates, E. A., Johnson, M. H., Karmiloff-Smith, A., Parisi, D., \& Plunkett, K. (1996). Rethinking innateness: A connectionist perspective on development. Cambridge, MA: MIT Press, Bradford Books.

Flores d'Arcais, G., Schreuder, R., \& Glazenborg, G. (1985). Semantic activation during recognition or referential words. Psychological Research, 47, 39-49.

Frith, C. (2000). The role of dorsolateral prefrontal cortex in the selection of action as revealed by functional imaging. In S. Monsell \& J. S. Driver (Eds.), Control of cognitive processes: Attention and performance XVIII (pp. 549-565). Cambridge, MA: MIT Press.

Gainotti, G. (2000). What the locus of brain lesion tells us about the nature of the cognitive defect underlying category-specific disorders: A review. Cortex, 36, 539-559. doi:10.1016/S0010-9452(08) 70537-9

Garcea, F. E., \& Mahon, B. Z. (2012). What is in a tool concept? Dissociating manipulation knowledge from function knowledge. Memory \& Cognition, 40, 1303-1313.

Gotts, S. J., Milleville, S. C., \& Martin, A. (2015). Object identification leads to a conceptual broadening of object representations in lateral prefrontal cortex. Neuropsychologia, 76, 62-78. doi:10.1016/j. neuropsychologia.2014.10.041
Hauk, O., \& Tschentscher, N. (2013). The body of evidence: What can neuroscience tell us about embodied semantics? Frontiers in Psychology, 4, 50. doi:10.3389/fpsyg.2013.00050

Herrera, E., Rodríguez-Ferreiro, J., \& Cuetos, F. (2012). The effect of motion content in action naming by Parkinson's disease patients. Cortex, 48, 900-904.

Hoenig, K., Muller, C., Herrnberger, B., Spitzer, M., Ehret, G., \& Kiefer, M. (2011). Neuroplasticity of semantic maps for musical instruments in professional musicians. NeuroImage, 56, 1714-1725.

Hoenig, K., Sim, E.-J., Bochev, V., Herrnberger, B., \& Kiefer, M. (2008). Conceptual flexibility in the human brain: Dynamic recruitment of semantic maps from visual, motion and motor-related areas. Journal of Cognitive Neuroscience, 20, 1799-1814. doi:10.1162/jocn.2008. 20123

Hsu, N. S., Kraemer, D. J. M., Oliver, R. T., Schlichting, M. L., \& Thompson-Schill, S. L. (2011). Color, context, and cognitive style: Variations in color knowledge retrieval as a function of task and subject variables. Journal of Cognitive Neuroscience, 29, 2544 2557.

Humphreys, G. W., \& Forde, E. M. (2001). Hierarchies, similarity, and interactivity in object recognition: "Category-specific" neuropsychological deficits. Behavioral and Brain Sciences, 24, 453-476. disc. $476-509$.

Kalénine, S., Mirman, D., Middleton, E. L., \& Buxbaum, L. J. (2012). Temporal dynamics of activation of thematic and functional knowledge during conceptual processing of manipulable artifacts. Journal of Experimental Psychology: Learning, Memory, and Cognition, 38, 1274-1295. doi:10.1037/a0027626

Kalénine, S., Shapiro, A. D., Flumini, A., Borghi, A. M., \& Buxbaum, L. J. (2014). Visual context modulates potentiation of grasp types during semantic object categorization. Psychonomic Bulletin \& Review, 21, 645-651. doi:10.3758/s13423-013-0536-7

Kan, I. P., Kable, J. W., Van Scoyoc, A., Chatterjee, A., \& ThompsonSchill, S. L. (2006). Fractionating the left frontal response to tools: Dissociable effects of motor experience and lexical competition. Journal of Cognitive Neuroscience, 18, 267-277.

Kan, I. P., \& Thompson-Schill, S. L. (2004a). Effect of name agreement on prefrontal activity during overt and covert picture naming. Cognitive, Affective, \& Behavioral Neuroscience, 4, 43-57. doi:10. 3758/CABN.4.1.43

Kan, I. P., \& Thompson-Schill, S. L. (2004b). Selection from perceptual and conceptual representations. Cognitive, Affective, \& Behavioral Neuroscience, 4, 466-482. doi:10.3758/CABN.4.4.466

Kiefer, M., \& Martens, U. (2010). Attentional sensitization of unconscious cognition: Task sets modulate subsequent masked semantic priming. Journal of Experimental Psychology: General, 139, 464489.

Kiefer, M., \& Pulvermüller, F. (2012). Conceptual representations in mind and brain: Theoretical developments, current evidence and future directions. Cortex, 48, 805-825. doi:10.1016/j.cortex.2011. 04.006

Kiefer, M., Sim, E.-J., Liebich, S., Hauk, O., \& Tanaka, J. (2007). Experience-dependent plasticity of conceptual representations in human sensory-motor areas. Journal of Cognitive Neuroscience, 19, $525-542$.

Kirby, J., Moore, P., \& Schofield, N. (1988). Verbal and visual learning styles. Contemporary Educational Psychology, 13, 169-184.

Kraemer, D. J., Rosenberg, L. M., \& Thompson-Schill, S. L. (2009). The neural correlates of visual and verbal cognitive styles. Journal of Neuroscience, 29, 3792-3798. doi:10.1523/JNEUROSCI.4635-08. 2009

Lebois, L. A. M., Wilson-Mendenhall, C. D., \& Barsalou, L. W. (2014). Are automatic conceptual cores the gold standard of semantic processing? The context-dependence of spatial meaning in grounded 
congruency effects. Cognitive Science. Advance online publication. doi:10.1111/cogs.12174

Lee, C.-L., Middleton, E. L., Mirman, D., Kalénine, S., \& Buxbaum, L. J. (2013). Incidental and context-responsive activation of structureand function-based action features during object identification. Journal of Experimental Psychology: Human Perception and Performance, 39, 257-270. doi:10.1037/a0027533

Lee, C.-L., Mirman, D., \& Buxbaum, L. J. (2014). Abnormal dynamics of activation of object use information in apraxia: Evidence from eyetracking. Neuropsychologia, 59, 13-26. doi:10.1016/j. neuropsychologia.2014.04.004

Lupyan, G., \& Mirman, D. (2013). Linking language and categorization: Evidence from aphasia. Cortex, 49, 1187-1194. doi:10.1016/j. cortex.2012.06.006

Lupyan, G., Mirman, D., Hamilton, R., \& Thompson-Schill, S. L. (2012). Categorization is modulated by transcranial direct current stimulation over left prefrontal cortex. Cognition, 124, 36-49. doi:10.1016/ j.cognition.2012.04.002

Lupyan, G., \& Thompson-Schill, S. L. (2012). The evocative power of words: Activation of concepts by verbal and nonverbal means. Journal of Experimental Psychology: General, 141, 170-186. doi: 10.1037/a0024904

Mahon, B. Z. (2015). What is embodied about cognition? Language, Cognition, and Neuroscience, 30, 420-429. doi:10.1080/ 23273798.2014.987791

Mahon, B. Z., \& Caramazza, A. (2008). A critical look at the embodied cognition hypothesis and a new proposal for grounding conceptual content. Journal of Physiology, 102, 59-70. doi:10.1016/j. jphysparis.2008.03.004

Malt, B. C., \& Johnson, E. C. (1992). Do artifact concepts have cores? Journal of Memory and Language, 31, 195-217. doi:10.1016/0749596X(92)90011-L

Martens, U., Ansorge, U., \& Kiefer, M. (2011). Controlling the unconscious: Attentional task sets modulate subliminal semantic and visuo-motor processes differentially. Psychological Science, 22, 282-291.

Masson, M. E. J., Bub, D. N., \& Breuer, A. T. (2011). Priming of reach and grasp actions by handled objects. Journal of Experimental Psychology: Human Perception and Performance, 37, 1470-1484.

McClelland, J. L., Rumelhart, D. E., \& the PDP Research Group. (1986). Parallel distributed processing: Explorations in the microstructure of cognition: Psychological and biological models (Vol. 2). Cambridge, MA: MIT Press.

McCloskey, M., \& Glucksberg, S. (1979). Decision processes in verifying category membership statements: Implications for models of semantic memory. Cognitive Psychology, 11, 1-37. doi:10.1016/ 0010-0285(79)90002-1

McRae, K., de Sa, V. R., \& Seidenberg, M. S. (1997). On the nature and scope of featural representations of word meaning. Journal of Experimental Psychology: General, 126, 99-130. doi:10.1037/ 0096-3445.126.2.99

Mechelli, A., Price, C. J., Friston, K. J., \& Ishai, A. (2004). Where bottom-up meets top-down: Neuronal interactions during perception and imagery. Cerebral Cortex, 14, 1256-1265.

Meyer, D. E., \& Schvaneveldt, R. W. (1971). Facilitation in recognizing pairs of words: Evidence of a dependence between retrieval operations. Journal of Experimental Psychology, 90, 227-234. doi:10. 1037/h0031564

Miller, E. K., \& Cohen, J. D. (2001). An integrative theory of prefrontal cortex function. Annual Review of Neuroscience, 24, 167-202. doi: 10.1146/annurev.neuro.24.1.167

Moss, H. E., McCormick, S. F., \& Tyler, L. K. (1997). The time course of activation of semantic information during spoken word recognition. Language and Cognitive Processes, 12, 695-731.
Mummery, C. J., Patterson, K., Hodges, J. R., \& Price, C. J. (1998). Functional neuroanatomy of the semantic system: Divisible by what? Journal of Cognitive Neuroscience, 10, 766-777.

Musz, E., \& Thompson-Schill, S. L. (2015). Semantic variability predicts neural variability of object concepts. Neuropsychologia, 76, 41-51. doi:10.1016/j.neuropsychologia.2014.11.029

Myung, J.-Y., Blumstein, S. E., Yee, E., Sedivy, J. C., Thompson-Schill, S. L., \& Buxbaum, L. J. (2010). Impaired access to manipulation features in apraxia: Evidence from eyetracking and semantic judgment tasks. Brain and Language, 112, 101-112. doi:10.1016/j. bandl.2009.12.003

Negri, G. A. L., Rumiati, R. I., Zadini, A., Ukmar, M., Mahon, B. Z., \& Caramazza, A. (2007). What is the role of motor simulation in action and object recognition? Evidence from apraxia. Cognitive Neuropsychology, 24, 795-816.

Noppeney, U., Price, C. J., Friston, K. J., \& Penny, W. D. (2006). Two distinct neural mechanisms for category-selective responses. Cerebral Cortex, 16, 437-445.

Oliver, R. T., Geiger, E. J., Lewandowski, B. C., \& Thompson-Schill, S. L. (2009). Remembrance of things touched: How sensorimotor experience affects the neural instantiation of object form. Neuropsychologia, 47, 239-247. doi:10.1016/j.neuropsychologia. 2008.07.027

Oliver, R. T., Parsons, M. A., \& Thompson-Schill, S. L. (2008, April). Hands on learning: Variations in sensorimotor experience alter the cortical response to newly learned objects. Poster presented at the annual meeting of the Cognitive Neuroscience Society, San Francisco, CA.

Patterson, K., Nestor, P. J., \& Rogers, T. T. (2007). Where do you know what you know? The representation of semantic knowledge in the human brain. Nature Reviews Neuroscience, 8, 976-987. doi:10. 1038/nrn2277

Pecher, D., Zeelenberg, R., \& Raaijmakers, J. G. W. (1998). Does pizza prime coin? Perceptual priming in lexical decision and pronunciation. Journal of Memory and Language, 38, 401-418. doi:10.1006/ jmla.1997.2557

Phillips, J. A., Noppeney, U., Humphreys, G. W., \& Price, C. J. (2002). Can segregation within the semantic system account for category specific deficits? Brain, 125, 2067-2080.

Pobric, G., Jefferies, E., \& Lambon Ralph, M. A. (2010). Categoryspecific versus category-general semantic impairment induced by transcranial magnetic stimulation. Current Biology, 20, 964-968. doi:10.1016/j.cub.2010.03.070

Polyn, S. M., Norman, K. A., \& Kahana, M. J. (2009). Task context and organization in free recall. Neuropsychologia, 47, 2158-2163.

Posner, M. I., \& Snyder, C. R. (1975). Attention and cognitive control. In R. L. Solso (Ed.), Information processing and cognition: The Loyola symposium (pp. 55-85). Hillsdale, NJ: Erlbaum.

Prinz, J. J. (2002). Furnishing the mind: Concepts and their perceptual basis. Cambridge, MA: MIT Press.

Pulvermüller, F. (1999). Words in the brain's language. Behavioral and Brain Sciences, 22, 253-279. disc. 280-336.

Rodríguez-Ferreiro, J., Gennari, S. P., Davies, R., \& Cuetos, F. (2011). Neural correlates of abstract verb processing. Journal of Cognitive Neuroscience, 23, 106-118. doi:10.1162/jocn.2010.21414

Rogers, T. T., Hocking, J., Mechelli, A., Patterson, K., \& Price, C. (2005). Fusiform activation to animals is driven by the process, not the stimulus. Journal of Cognitive Neuroscience, 17, 434-445. doi:10. 1162/0898929053279531

Rogers, T. T., \& McClelland, J. L. (2004). Semantic cognition: A parallel distributed processing approach. Cambridge, MA: MIT Press.

Rogers, T. T., \& Patterson, K. (2007). Object categorization: Reversals and explanations of the basic-level advantage. Journal of Experimental Psychology: General, 136, 451-469. doi:10.1037/ 0096-3445.136.3.451 
Schacter, D. L. (1995). Memory distortion: History and current status. In D. L. Schacter, J. T. Coyle, \& Harvard Center for the Study of Mind, Brain, and Behavior (Eds.), Memory distortion: How minds, brains, and societies reconstruct the past (pp. 1-43). Cambridge, MA: Harvard University Press.

Schreuder, R., Flores d'Arcais, G. B., \& Glazenborg, G. (1984). Effects of perceptual and conceptual similarity in semantic priming. Psychological Research, 45, 339-354.

Schyns, P. G., Goldstone, R. L., \& Thibaut, J.-P. (1998). The development of features in object concepts. Behavioral and Brain Sciences, 21, 1-17. doi:10.1017/S0140525X98520109. disc. 17-54.

Spivey, M. J. (2007). The continuity of mind. New York, NY: Oxford University Press.

Swinney, D. A. (1979). Lexical access during sentence comprehension: (Re)consideration of context effects. Journal of Verbal Learning and Verbal Behavior, 18, 645-659.

Tabossi, P. (1988). Effects of context on the immediate interpretation of unambiguous nouns. Journal of Experimental Psychology: Learning, Memory, and Cognition, 14, 153-162. doi:10.1037/ 0278-7393.14.1.153

Tanenhaus, M. K., Spivey-Knowlton, M. J., Eberhard, K. M., \& Sedivy, J. C. (1995). Integration of visual and linguistic information in spoken language comprehension. Science, 268, 1632-1634. doi:10. 1126/science. 7777863

Taylor, L. J., \& Zwaan, R. A. (2009). Action in cognition: The case of language. Language and Cognition, 1, 45-58.

Thompson-Schill, S. L. (2003). Neuroimaging studies of semantic memory: Inferring how from where. Neuropsychologia, 41, 280-292.

Thompson-Schill, S. L., Aguirre, G. K., D’Esposito, M., \& Farah, M. J. (1999). A neural basis for category and modality specificity of semantic knowledge. Neuropsychologia, 37, 671-676. doi:10.1016/ S0028-3932(98)00126-2

Thompson-Schill, S. L., Bedny, M., \& Goldberg, R. F. (2005). The frontal lobes and the regulation of mental activity. Current Opinion in Neurobiology, 15, 219-224.

Trumpp, N. M., Kliese, D., Hoenig, K., Haarmeier, T., \& Kiefer, M. (2013). Losing the sound of concepts: Damage to auditory association cortex impairs the processing of sound-related concepts. Cortex, 49, 474-486.

Tucker, M., \& Ellis, R. (1998). On the relations between seen objects and components of potential actions. Journal of Experimental Psychology: Human Perception and Performance, 24, 830-846. doi:10.1037/0096-1523.24.3.830

Tyler, L. K., \& Moss, H. E. (2001). Towards a distributed account of conceptual knowledge. Trends in Cognitive Sciences, 5, 244-252. doi:10.1016/S1364-6613(00)01651-X

van Dam, W. O., Rueschemeyer, S.-A., Lindemann, O., \& Bekkering, H. (2010). Context effects in embodied lexical-semantic processing. Frontiers in Psychology, 1, 150. doi:10.3389/fpsyg.2010.00150 van Dam, W. O., van Dijk, M., Bekkering, H., \& Rueschemeyer, S.-A. (2012). Flexibility in embodied lexical-semantic representations. Human Brain Mapping, 33, 2322-2333.

van Dantzig, S., Pecher, D., Zeelenberg, R., \& Barsalou, L. W. (2008). Perceptual processing affects conceptual processing. Cognitive Science, 32, 579-590. doi:10.1080/03640210802035365

Vermeulen, N., Corneille, O., \& Niedenthal, P. M. (2008). Sensory load incurs conceptual processing costs. Cognition, 109, 287-294.

Vigliocco, G., Vinson, D. P., Lewis, W., \& Garrett, M. F. (2004). Representing the meanings of object and action words: The featural and unitary semantic space hypothesis. Cognitive Psychology, 48, 422-488. doi:10.1016/j.cogpsych.2003.09.001

Weisberg, J., Turennout, M., \& Martin, A. (2007). A neural system for learning about object function. Cerebral Cortex, 17, 513-521. doi: 10.1093/cercor/bhj176

Willems, R. M., \& Casasanto, D. (2011). Flexibility in embodied language understanding. Frontiers in Psychology, 2(116), 1-11. doi: 10.3389/fpsyg.2011.00116

Willems, R. M., \& Francken, J. C. (2012). Embodied cognition: Taking the next step. Frontiers in Psychology, 3, 582. doi:10.3389/fpsyg. 2012.00582

Willems, R. M., Hagoort, P., \& Casasanto, D. (2010). Body-specific representations of action verbs: Neural evidence from right- and left-handers. Psychological Science, 21, 67-74. doi:10.1177/ 0956797609354072

Witt, J. K., Kemmerer, D., Linkenauger, S. A., \& Culham, J. (2010). A functional role for motor simulation in identifying tools. Psychological Science, 21, 1215-1219. doi:10.1177/ 0956797610378307

Yee, E., Ahmed, S. Z., \& Thompson-Schill, S. L. (2012). Colorless green ideas (can) prime furiously. Psychological Science, 23, 364-369. doi:10.1177/0956797611430691

Yee, E., Chrysikou, E. G., Hoffman, E., \& Thompson-Schill, S. L. (2013a). Manual experience shapes object representation. Psychological Science, 24, 909-919. doi:10.1177/ 0956797612464658

Yee, E., Chrysikou, E. G., \& Thompson-Schill, S. L. (2013b). The cognitive neuroscience of semantic memory. In K. Ochsner \& S. Kosslyn (Eds.), The Oxford handbook of cognitive neuroscience, Vol. 1: Core topics (pp. 353-374). Oxford, UK: Oxford University Press.

Yee, E., Huffstetler, S., \& Thompson-Schill, S. L. (2011). Function follows form: Activation of shape and function features during object identification. Journal of Experimental Psychology: General, 140, 348-363. doi:10.1037/a0022840

Zwaan, R. A., Stanfield, R. A., \& Yaxley, R. H. (2002). Language comprehenders mentally represent the shapes of objects. Psychological Science, 13, 168-171. doi:10.1111/1467-9280.00430 